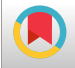

\title{
Prevalence of Malnutrition Amongst Adult Patients Admitted to Emam Ali Hospital in Zahedan, 2016
}

\author{
Farzaneh Montazerifar ${ }^{1,{ }^{*}}$, Mansour Karajibani ${ }^{2,{ }^{* *}}$, Ali Reza Dashipour ${ }^{3}$, Nadia Nouri $^{4}$, Zohreh \\ Khaksefidi ${ }^{4}$ and Elaheh Najjari ${ }^{4}$ \\ ${ }^{1}$ Pregnancy Health Research Center, Department of Nutrition, School of Medicine, Zahedan University of Medical Sciences, Zahedan, Iran \\ ${ }^{2}$ Health Promotion Research Center, Department of Nutrition, School of Medicine, Zahedan University of Medical Sciences, Zahedan, Iran \\ ${ }^{3}$ Department of Nutrition and Food Sciences, School of Medicine, Zahedan University of Medical Sciences, Zahedan, Iran \\ ${ }^{4}$ Department of Nutrition, School of Medicine, Zahedan University of Medical Sciences, Zahedan, Iran \\ "Corresponding author: Associate Professor of Nutrition, Department of Nutrition, School of Medicine, Zahedan University of Medical Sciences, Zahedan, Iran. Tel: \\ +98-9155416903, Fax: +98-5433295728, Email: fmontazerifar@gmail.com \\ "* Corresponding author: Associate Professor, Health Promotion Research Center, Department of Nutrition, School of Medicine, Zahedan University of Medical Sciences \\ Zahedan, Iran. Email: mkarajibani@yahoo.com
}

Received 2017 May 13; Revised 2017 October 24; Accepted 2018 March 18.

\begin{abstract}
Background: Malnutrition in hospitalized patients is a major health problem, which has devastating effects on the healthcare system.

Objectives: The main objective of this study was to estimate the prevalence of malnutrition in adult patients on admission to the hospital.

Methods: In a cross-sectional study, 450 adult patients (mean aged $44.6 \pm 15.3$ years), on admission to the hospital, were selected from a general teaching hospital in Zahedan, Iran. The assessment of malnutrition was performed based on the anthropometric parameters and using of malnutrition universal screening tool (MUST). In addition, serum albumin level was also measured.

Results: The prevalence of malnutrition, according to the MUST criteria, was $40 \%$. The prevalence in age group $>50$ years old was markedly higher compared to those $<30$ years $(56.2 \%$ vs. $37.7 \%$, OR $=1.6 ; 95 \% \mathrm{CI}$ : $0.71-2.5 ; \mathrm{P}<0.01)$. The patients with mid upper arm circumference $(\mathrm{MUAC})<5$ th $(53.6 \%$ vs. $31.9 \%, \mathrm{OR}=2.6 ; 95 \% \mathrm{CI}: 1.93-3.47 ; \mathrm{P}=0.009)$ and also those with serum albumin $<3 \mathrm{~g} / \mathrm{dL}(71.9$ $\%$ vs $50 \%, \mathrm{OR}=1.6 ; 95 \% \mathrm{CI}: 0.93-2.2 ; \mathrm{P}=0.047)$ had significantly higher odds of malnutrition as compared to normal patients. The highest prevalence of malnutrition was observed in the nephrology ward (48\%).

Conclusions: The findings showed that the prevalence of malnutrition in patients admitted is relatively high. Since, nutritional status may be deteriorated during hospital stay, it is suggested that nutritional screening by a simple procedure are applied at admission to hospital.
\end{abstract}

Keywords: Prevalence, Malnutrition, Hospital Admission, Adult Patients

\section{Background}

Malnutrition in hospitalized adults is a worldwide problem, which is often not considered as a priority adequate medical $(1,2)$. Depending on the definition and diagnosis criteria as well as patients' population, the prevalence of malnutrition in hospitalized patients is estimated to be between $30 \%-50 \%(1,3,4)$, which is more common among older patients (5). In long-term care facilities, the rate of malnutrition has been reported as $85 \%$ (3). In Iran, few studies have been done on the prevalence of malnutrition in hospitalized patients and findings of these studies suggest a high prevalence of malnutrition on admission to the hospital (5-9). Malnutrition in hospitalized patients is the result of some factors including extreme loss and/or insufficient intake of calorie, protein, vitamins, minerals and other nutrients, elevated requirement, and/or altered utilization of nutrients $(2,10)$. As well, the changing in diet composition and fasting for examinations and other procedures can cause a loss of appetite or food intake disorders, leading to a deterioration of nutritional status during the time of hospitalization(11). Several studies have reported that malnutrition in hospitalized patients increases the length of hospital stay and healthcare costs, decreases quality of life (10), and contributes to increased morbidity and mortality in patients $(10,12)$. On the other hand, it is associated with negative outcomes for patients, including complications such as cachexia (characterized by severe loss weight, fat and muscle and high catabolism of protein), inadequate intake of nutrients (un- 
dernutrition), malabsorption, infection, impaired wound healing, or a combination of aforementioned factors $(2,13$, 14). The numerous published studies have shown that malnutrition is an important health problem with poor diagnosis. Thus, the screening and assessing patients' nutritional status in hospital using of simple, fast, and accurate tools contributes to rapidly identifying patients at risk of malnutrition and their treatment $(2,15)$. There are numerous assessment tools for screening, identifying risk, and diagnose of malnutrition. Malnutrition universal screening tool (MUST) is a valid and useful screening tool to predict protein-energy malnutrition in adults, which is usually used in several countries with different health care settings $(16,17)$. The criteria for diagnosis of malnutrition by MUST includes current weight status using BMI, unintentional weight loss in the previous 3 - 6 months $(2,5,11,16)$, and acute disease effect (16). MUST detects both undernutrition and over nutrition in adults, and has been designed for use in multiple settings including hospitals and nursing homes (2).

\section{Objectives}

Since, the prevalence of malnutrition in the Zahedan hospitals has not been reported until now, the aim of this study was to evaluate the prevalence of malnutrition among hospitalized adult patients at the time of admission in a general training hospital in Zahedan, which is located in the south-east of Iran.

\section{Methods}

In a cross-sectional study, 450 adult patients (mean aged $44.6 \pm 15.3$ years; ranged 18 - 77 years), were selected from Emam Ali Hospital in Zahedan, which is located in the south-east of Iran. Sample size was statistically estimated based on the prevalence of malnutrition of about 45\% [5] and by the following formula: $\mathrm{n}=\mathrm{z}^{2} \mathrm{pq} / \mathrm{d}^{2}$.

Data collection was carried out between June 2015 to May 2016. The patients were recruited within 72 hours of admission to the hospital from different wards including surgery, nephrology, gastroenterology, and CCU. The exclusion criteria included age less than 18 years, pregnancy, unable to complete the questionnaire, psychiatric or communication difficulties, physical incapacity, and also hospitalized patients in the intensive care unit (ICU). A general questionnaire to evaluate personal and sociodemographic data including age, sex, educational level, hospitalized readmission in the previous six months, and hospitalization ward were completed for each patient. The assessments were performed by three well-trained nutrition students under the supervision of a nutritionist (study author).

\subsection{Nutritional Assessment}

Malnutrition status was assessed by universal screening tool (MUST) and anthropometric measurements (e.g., weight, height, BMI, and mid-upper arm circumference), nutritional data including appetite assessment and gastrointestinal problems (e.g., diarrhea, decreased appetite, nausea and vomiting, deglutition disorder), and biochemical test results detecting low levels of serum albumin.

The recent weight and height of patients were measured using Seca platform scale, Germany, and stadiometer, respectively. Usual weight of patients was asked in a period of three to six months. To calculate the body mass index (BMI), recent weight (kg) was divided by height squared $\left(\mathrm{m}^{2}\right)$. The percentage unintentional weight loss in the previous three to six months was calculated from patients' reports and scored according to the MUST criteria (16).

In this study, patients were classified into three malnutrition risk categories (low, medium and high) as follows: Patients who had BMI of $<18.5 \mathrm{~kg} / \mathrm{m}^{2}$ and a history of unintentional weight loss of $>10 \%$ the previous three to six months was considered as high risk; BMI $18.5-20 \mathrm{~kg} / \mathrm{m}^{2}$ and a history of unintentional weight loss $5 \%-10 \%$ in a period of three to six months as medium risk; and BMI $>20 \mathrm{~kg} / \mathrm{m}^{2}$ and unintentional weight loss $<5 \%$ in a period of three to six months was considered as low risk $(5,16)$. Overall, the results were reported as low risk and high risk (medium + high risk was considered as high risk).

The mid-upper arm circumference (MUAC) was measured with a non-stretchable tape measure at the midpoint of the right upper arm between the olecranon of the ulna and the acromion of the scapula. The values of MUAC were analyzed based on percentile tables (18). In addition, serum albumin was assessed in 184 of the patients and measured by photometric method.

All participants gave oral informed consent. The protocol of study was approved by the Ethics Research Committee of Zahedan University of Medical Sciences. Code No: IR. ZAUMS. REC.1394.28.

\subsection{Statistical Analysis}

Data were analyzed using SPSS statistical software package program (version 21 for windows, Chicago, USA). The results were expressed as mean \pm standard deviation with range, and odds ratio and 95\% confidence interval (CI), as appropriate. In order to evaluate the risk of malnutrition, the multiple logistic regression was performed to determine risk-adjusted factors. Data were compared by the Chi-square test. $\mathrm{P}<0.05$ was considered statistically significant. 


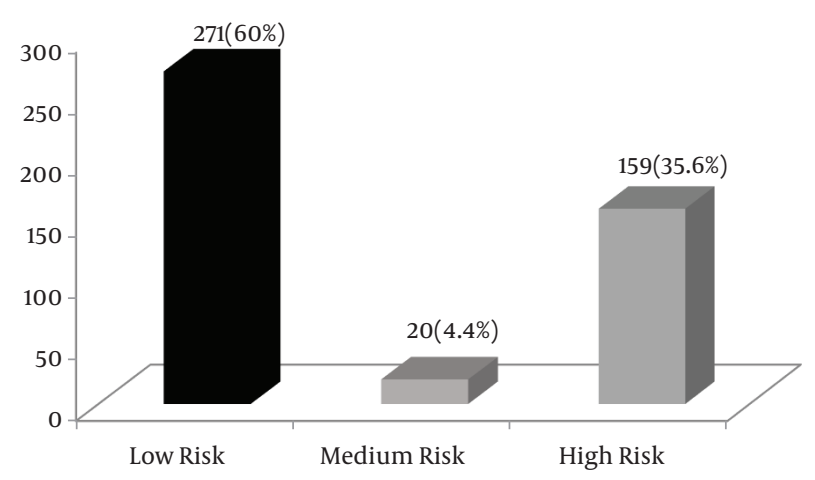

Figure 1. Prevalence of malnutrition according to MUST classification

\section{Results}

Demographic characteristics and nutritional parameters of 450 hospitalized patients on admission are demonstrated in Table1. The study population included 221(49.1\%) men and 229 (50.9\%) women aged 18 to 77 years old. Most patients $(22.9 \%$ and $20.7 \%$ ) had nausea, vomiting, and diarrhea, respectively. More than half of the patients (54.9\%) had loss of appetite. A total of $27.3 \%$ had a low BMI alone $\left(<18.5 \mathrm{~kg} / \mathrm{m}^{2}\right)$, and $56.2 \%$ a history of unintentional weight loss alone ( $>10 \%) ; 43.1 \%$ of patients had MUAC $<5$ th. The serum albumin levels were measure in 184 subjects. Of those who assessed serum albumin, $48.4 \%$ had low levels $(<3 \mathrm{~g} / \mathrm{dL})$.

As shown in Figure 1, the prevalence of malnutrition (medium risk + high risk) was 40\% (4.4\% medium risk, 35.6 $\%$ high risk), and $60 \%$ were at low risk of malnutrition.

Table 2 demonstrates the prevalence of malnutrition using 2-category classification of MUST (low and high risk) association with demographic variables. In addition, the multiple regression models are represented in Table 3. The results showed that malnutrition prevalence was similar among both men and women $(\mathrm{P}=0.82)$. The patients $>50$ years old had a higher rate of malnutrition compared to those $<30$ years $(56.2 \%$ vs. $37.7 \%$; OR $=1.6 ; 95 \% \mathrm{CI}$ : $0.71-2.5$; $\mathrm{P}<0.01)$. No association was found between malnutrition and the levels of education $(\mathrm{P}=0.06)$.

Among the different wards of the hospital, the highest rate of malnutrition was found in the nephrology ward (48\%); however, there was no significant difference between the wards $(\mathrm{P}=0.08)$. The patients with different gastrointestinal disorders had a slightly higher rate of malnutrition, however, they did not have a significant difference compared to the other patients ( $40.8 \%$ vs. $37.5 \%$; $\mathrm{P}=0.17$ ).

The patients with loss of appetite had a higher rate of malnutrition compared to those who had no loss of appetite (43.9\% vs. $34.8 \%)$.

\begin{tabular}{|c|c|}
\hline Parameters & Value $^{a}$ \\
\hline $\operatorname{Age}(y)$ & $44.6 \pm 15.3$ \\
\hline Recent weight (kg) & $60.7 \pm 13.7$ \\
\hline Unintentional weight loss & $64.8 \pm 14.9$ \\
\hline Height $(\mathrm{cm})$ & $167.5 \pm 6.5$ \\
\hline $\operatorname{BMI}\left(\mathbf{k g} / \mathbf{m}^{2}\right)$ & $21.6 \pm 4.4$ \\
\hline $\operatorname{MUAC}(\mathbf{c m})$ & $26 \pm 4.5$ \\
\hline Serum albumin (g/dL) & $3.2 \pm 0.93$ \\
\hline Hospitalized readmission (times) (range) & $3(1-15)$ \\
\hline \multicolumn{2}{|l|}{ Gastrointestinal disorders } \\
\hline Nausea and vomiting & $103(22.9)$ \\
\hline Diarrhea & $92(20.4)$ \\
\hline Constipation & $44(9.8)$ \\
\hline Difficulty chewing and deglutition disorder & $50(11.1)$ \\
\hline None & $161(35.8)$ \\
\hline \multicolumn{2}{|l|}{ Loss of appetite } \\
\hline Yes & $247(54.9)$ \\
\hline No & $203(45.1)$ \\
\hline \multicolumn{2}{|l|}{ BMI $\left(\mathbf{K g} / \mathbf{m}^{2}\right)$} \\
\hline$<18.5$ & $123(27.3)$ \\
\hline $18.5-20$ & $60(13.3)$ \\
\hline$>20$ & $267(59.4)$ \\
\hline \multicolumn{2}{|l|}{ History of weight loss } \\
\hline$>10 \%$ & $253(56.2)$ \\
\hline $5 \%-10 \%$ & $2(0.4)$ \\
\hline$<5 \%$ & $195(43.4)$ \\
\hline \multicolumn{2}{|l|}{ MUAC (percentile) } \\
\hline$<5$ th & $194(43.1)$ \\
\hline 5th - 10th & $49(10.9)$ \\
\hline$>10$ th & $207(46)$ \\
\hline \multicolumn{2}{|l|}{ Albumin (g/dL) } \\
\hline$<3$ & $89(48.4)$ \\
\hline $3-3.5$ & $29(15.8)$ \\
\hline$>3.5$ & $66(35.8)$ \\
\hline
\end{tabular}

Abbreviations: BMI, body mass index; MUAC, mid upper arm circumference. ${ }^{\mathrm{a}}$ Values are expressed as mean $\pm \mathrm{SD}$ or No. (\%).

In the regression model, the patients with low $\mathrm{MUAC}(<$ 5th) (53.6\% vs. 31.9\%; OR = 2.6; 95\% CI: $1.93-3.47 ; \mathrm{P}=0.009)$, and also the patients with low levels of serum albumin $(<$ $3 \mathrm{~g} / \mathrm{dL})(71.9 \%$ vs. 50\%; OR = 1.6; 95\% CI: $0.93-2.2 ; \mathrm{P}=0.047)$ had significantly higher rate of malnutrition as compared to normal patients. 


\begin{tabular}{|c|c|c|c|}
\hline Malnutrition Parameters & Low Risk & High Risk & P Value \\
\hline Sex & & & 0.82 \\
\hline Male & $141(63.8)$ & $80(36.2)$ & \\
\hline Female & $130(56.8)$ & $99(43.2)$ & \\
\hline Age $(y)$ & & & 0.01 \\
\hline$\leq 30$ & $66(62.3)$ & $40(37.7)$ & \\
\hline $31-50$ & $124(78)$ & $35(22)$ & \\
\hline$>50$ & $81(43.8)$ & $104(56.2)$ & \\
\hline Education & & & 0.06 \\
\hline Under diploma & $194(60.1)$ & $129(39.9)$ & \\
\hline Diploma and over & $77(60.6)$ & $50(39.4)$ & \\
\hline Kind of ward & & & 0.08 \\
\hline Nephrology & $79(52)$ & $73(48)$ & \\
\hline Surgery & $38(62.3)$ & $23(37.7)$ & \\
\hline Gastroenterology & $143(63.8)$ & $81(36.2)$ & \\
\hline $\mathrm{CCU}$ & $11(84.6)$ & $2(15.4)$ & \\
\hline Gastrointestinal disorders & & & 0.17 \\
\hline Yes & $171(59.2)$ & $118(40.8)$ & \\
\hline No & $100(62.5)$ & $61(37.5)$ & \\
\hline Decreased appetite & & & 0.058 \\
\hline Yes & $138(56.1)$ & $109(43.9)$ & \\
\hline No & $133(65.2)$ & $70(34.8)$ & \\
\hline Serum albumin (g/dL) & & & 0.05 \\
\hline$<3$ & $25(28.1)$ & $64(71.9)$ & \\
\hline $3-3.5$ & $15(51.7)$ & $14(49.3)$ & \\
\hline$>3.5$ & $33(50)$ & $33(50)$ & \\
\hline MUAC (percentile) & & & 0.01 \\
\hline$<5$ th & $90(46.4)$ & $104(53.6)$ & \\
\hline $5-10$ th & $40(81.6)$ & $9(18.4)$ & \\
\hline$>10$ th & $141(68.1)$ & $66(31.9)$ & \\
\hline Hospitalized readmission & & & 0.048 \\
\hline No-hospitalized & $99(62.3)$ & $60(37.7)$ & \\
\hline$\geq 1$ & $172(49.1)$ & $119(50.9)$ & \\
\hline
\end{tabular}

bbreviation: MUAC, mid upper arm circumference.

${ }^{\text {a }}$ Values are expressed as No. (\%).

In addition, the patients with $\geq 1$ hospitalized readmission had higher rate of malnutrition in a regression model compared to subjects who had no history of hospitalization in the previous 6 months (50.9\% vs. 37.7\%; OR= 2.6; 95\%; CI: 1.3 - 3.95; $\mathrm{P}=0.05$ ).

\section{Discussion}

Malnutrition is a common complication in acute care hospital patients and is related to poor outcomes. Therefore, the evaluation of nutritional status on admission to hospital is necessary to identify the patients at risk of malnutrition. A high prevalence of malnutrition in the hospital setting between $20 \%$ and $50 \%(2,14,19)$, a range of $19 \%-60 \%$ in inpatients $(20,21)$, and $30 \%$ in outpatients has been documented in the literature (20). These differences could be related to the definition and criteria used for malnutrition diagnosis (14). Thus, the implementation of nutrition care programs in hospitals for identifying risk and diagnosis of malnutrition and its treatment has been recommended (15). In Iran, nutrition screening upon admission in the hospital is recently compulsory. However, few reports have been published on the prevalence of malnutrition at admission to hospital (6-8). Some studies suggest a high prevalence of malnutrition with different tools (4-9). MUST, as a simple screening tool with good validity (sensitivity $80.6 \%$, specificity $98.7 \%$ ) and almost perfect agreement $(\mathrm{k}=0.81)(22)$ with a combined index (BMI and weight-loss), was considered in some studies $(2,5,16,22)$.

In the present study, nutritional screening using MUST criteria showed that about two-fifths of patients had malnutrition, in which the majority of them were identified at high risk. This rate was greater than the rate reported in the Garcia et al. (17) study, and other studies that had used a diverse tool for evaluation of malnutrition in hospitalized patients (19). Stratton et al. (16) also reported that according to the MUST criteria, more than half of the hospitalized patients were at risk of malnutrition.

The rate of malnutrition was markedly higher according to BMI and history of unintentional weight loss in the previous three to six months alone. This rate was similar to the results of Foadoddini et al. (5), which was more than the rates reported by the study of Edington et al. (23).

Physiologically, malnutrition leads to decreased whole body protein concentrations and muscle and fat mass (24). Since, BMI alone is not a sensitive indicator to determine the fat and protein depletion, the measurement of midupper arm circumference "as a nutritional assessment tool to check for signs of muscle wasting" and serum albumin levels along with BMI accurately estimates body muscle mass and can increase the accuracy of malnutrition diagnosis $(4,24)$.

Therefore, in this study, in addition to the evaluation of BMI and history of unintentional weight loss, to determine the malnutrition, mid-upper arm circumference (MUAC) and serum albumin levels were also assessed. The findings showed that half of the patients with MUAC $<5$ th and more than two thirds of those with serum levels of albu$\min <3 \mathrm{~g} / \mathrm{dL}$ were at a high risk of malnutrition. This represents an alarming situation, which malnutrition is com- 


\begin{tabular}{|c|c|c|c|c|}
\hline Model & OR (CI 95\%) & Standardized Coefficients & $\mathbf{t}$ & P Value \\
\hline Variables & & Beta & & \\
\hline Age & $1.6(0.71-2.5)$ & 0.292 & 2.621 & 0.01 \\
\hline MUAC & $2.6(1.93-3.47)$ & 0.891 & 3.96 & 0.009 \\
\hline Hospitalized readmission & $2.6(1.3-3.95)$ & 0.704 & 3.760 & 0.05 \\
\hline
\end{tabular}

Abbreviation: MUAC, mid upper arm circumference.

mon among hospitalized patients on admission, especially in the elderly, and in the absence of diagnosis, it can influence the treatment of patients, medical costs, the longer hospital stay, and mortality (11). .

It was observed that between factors investigated, MUAC $<5$ th and hospitalized readmission $\geq 1$, with a risk more than 2.5 fold, were more powerful predictors of malnutrition risk. In some studies, gender was a factor correlated to malnutrition, and the frequency observed in men was more than women $(4,5,11)$. The other studies revealed that weight loss during hospitalization was higher in women than in men $(6,25)$. However, there was no significant gender difference in the risk of malnutrition in the present study. It was in agreement to the previous studies $(19,26)$.

Some studies have shown that the prevalence of malnutrition increases with age $(5,14,27)$. In a study, the prevalence in age group $<30$ years was more than other age groups, which might be related to the indicator of nutritional status in different studies (4). Our findings showed that nearly half of the patients older than 50 years were at a high risk of malnutrition. The high prevalence of malnutrition in elderly patients can be related to an underlying disease, depression, inadequate nutrients intake, economic problems, and dental or chewing disorders (28). It has been reported that gastrointestinal disorders may cause loss of appetite or impaired food intake, and increase the risk of malnutrition $(4,11,29)$. In addition, a significant relationship between malnutrition, with difficulty chewing and deglutition disorders, decreased appetite, nausea, and vomiting was shown in previous studies $(11,29)$. In the present study, more than half of the patients had some gastrointestinal disorders. Among the disorders investigated (nausea and vomiting, diarrhea, constipation, difficulty chewing, and deglutition disorder), the most common ones were nausea, vomiting, and diarrhea. Of the total patients with gastrointestinal complaints, almost one third of them were at high risk of malnutrition, however, it was not statistically significant. The decreased appetite is also considered as an important variable associated with malnutrition $(11,30)$, which may indirectly evaluate dietary intake(11). The findings of our study showed that the patients with decreased appetite had nearly 1.2 fold higher risk of malnutrition. With regard to the findings, an adequate nutritional support in order to improve clinical outcome in these patients is recommended. Our findings demonstrated that the malnutrition risk was higher among patients admitted in the nephrology, surgery, and gastroenterology wards. In a study carried out by Foadoddini et al. (5), high prevalence was shown in the surgery, infectious, and CCU wards. In another study, the highest prevalence of malnutrition was observed in geriatric, oncology, and gastroenterology departments (14). The greater prevalence in surgery compared to oncology ward was reported in the study of Garcia et al. (17). The prevalence of malnutrition in hospital admissions in different wards varies according to the criteria used, however, based on MUST, it is estimated that $20 \%$ - $60 \%$ of patients in the medical, surgical, geriatric, and orthopedic wards are to be affected (5). The rates reported in our research and previous studies (5, $14,17)$ were in the same range. However, in the absence of appropriate screening procedures, the patients at risk of malnutrition in various wards of hospital do not seem to be recognized and/or are not referred for treatment $(5,31)$.

In the present study, the chance of high risk of malnutrition in the patients with hospitalized readmission $\geq 1$ was significantly higher than 2.5 - fold compared to those not hospitalized. Similarly, a study conducted in Tehran showed that hospitalized readmission increased the malnutrition (4), suggesting the hospitalized readmission could be considered as a main contributor to malnutrition.

In our study, the levels of education did not have an association with malnutrition. However, in several studies $(14,28)$, the low level of education has been reported as a factor associated with malnutrition. In addition, several studies have also revealed that although the prevalence of moderate $(4)$ and severe $(4,5)$ malnutrition in patients with a high level of education were less than those with low education, no association was found between malnutrition and education. The high rate of malnutrition in people with low education levels can be linked to an inadequate awareness about the nutritional needs during disease, improper food habits, and culture. Further studies 
are needed to determine whether socioeconomic factors such as education levels are effective in malnutrition.

This study has some limitations including noncompliance of patients; lack of measurement of other anthropometric parameters (such as triceps skinfold (TSF), mid-arm muscle circumference (MAMC), and biochemical data (such as prealbumin, C-reactive protein (CRP), creatinine height index (CHI)); limited the number of hospital wards; thus the results could not be representative of patients information in other hospitals.

The strength of the study was the use of MUST' as a nutrition screening tool, which has predictive validity in elderly hospitalized patients, even in those who can't be weighted.

\subsection{Conclusions}

Overall, findings revealed that almost half of the patients admitted need nutritional supports. Since, nutritional status may be deteriorated during hospital stay, it is suggested that nutritional screening, by a simple procedure, are applied at admission to hospital.

\section{Acknowledgments}

The authors would like to thank all the subjects who kindly cooperated in this study.

\section{Footnotes}

Authors' Contribution: Study concept and design: Farzaneh Montazerifar; acquisition of data: Nadia Nouri, Zohreh Khaksefidi, Elaheh Najjari; analysis and interpretation of data: Farzaneh Montazerifar, Mansour Karajibani; drafting of the manuscript: Fazaneh Montazerifar; Critical revision of the manuscript for important intellectual content: Farzaneh Montazerifar, Mansour Karajibani, Ali Reza Dashipour; statistical analysis: Ali Reza Dashipour, Fazaneh Montazerifar; administrative, technical, and material support: Farzaneh Montazerifar; study supervision: Farzaneh Montazerifar, Mansour Karajibani. Farzaneh Montazerifar and Mansour Karajibani contributed equally to this work.

Funding/Support: This work was supported by the Research Deputy of Zahedan University of Medical Sciences, Zahedan, Iran (Protocol No and date: 7245: 19 April 2015).

\section{References}

1. Barker LA, Gout BS, Crowe TC. Hospital malnutrition: Prevalence, identification and impact on patients and the healthcare system. Int J Environ Res Public Health. 2011;8(2):514-27. doi: 10.3390/ijerph8020514. [PubMed: 21556200]. [PubMed Central: PMC3084475]
2. Cawood AL, Elia M, Sharp SK, Stratton RJ. Malnutrition self-screening by using MUST in hospital outpatients: Validity, reliability, and ease of use. Am J Clin Nutr. 2012;96(5):1000-7. doi: 10.3945/ajcn.112.037853. [PubMed: 23034963].

3. Malone A, Hamilton C. The Academy of Nutrition and Dietetics/the American Society for Parenteral and Enteral Nutrition consensus malnutrition characteristics: Application in practice. Nutr Clin Pract. 2013;28(6):639-50. doi: 10.1177/0884533613508435. [PubMed: 24177285].

4. Hosseinpour-Niazi S, Naderi Z, Hosseinpour-Niazi N, Delshad M, Mirmiran P, Azizi F. [Prevalence of malnutrition in hospitalized patients in Taleghani Hospital in Tehran]. J Gorgan Uni Med Sci. 2012;13(4):97106. Persian.

5. Foadoddini M, Raghebi SS, Farhadian A, Sharifzadeh G. [Prevalence of malnutrition in adults admitted to the hospitals affiliated to Birjand University of Medical Sciences in 2011]. J Birjand Uni Med Sci. 2014;21(2):219-27. Persian.

6. Dinmohammadi MR, Purmemary MH. [Nutrition markers in underhemodialysis patients in Zanjan Shahid Beheshti Hospital]. J Zanjan Univ Med Sci. 2002;10(2):41-5. Persian.

7. Hosseini S, Amirkalali B, Nayebi N, Heshmat R, Larijani B. Nutrition status of patients during hospitalization, Tehran, Iran. Nutr Clin Pract. 2006;21(5):518-21. doi: 10.1177/0115426506021005518. [PubMed: 16998150].

8. Ashabi A, Nozari B, Tabibi H, Mahdavi-Mazdeh M, Hedayati M, Houshiar Rad A. [Prevalence of protein-energy malnutrition and its various types in hemodialysis patients in Tehran, 2008]. Iran J Nutr Sci Food Technol. 2010;5(1):17-28. Persian.

9. Tabibi H, As'habi A, Heshmati BN, Mahdavi-Mazdeh M, Hedayati $M$. Prevalence of protein-energy wasting and its various types in Iranian hemodialysis patients: A new classification. Ren Fail. 2012;34(10):1200-5. doi: 10.3109/0886022X.2012.718710. [PubMed: 23002898].

10. White JV, Guenter P, Jensen G, Malone A, Schofield M, Academy Malnutrition Work G, et al. Consensus statement: Academy of Nutrition and Dietetics and American Society for Parenteral and Enteral Nutrition: Characteristics recommended for the identification and documentation of adult malnutrition (undernutrition). JPEN J Parenter Enteral Nutr. 2012;36(3):275-83. doi: 10.1177/0148607112440285. [PubMed: 22535923].

11. Aquino Rde $C$, Philippi ST. Identification of malnutrition risk factors in hospitalized patients. Rev Assoc Med Bras (1992). 2011;57(6):637-43. [PubMed: 22249542].

12. Holmes $S$. The effects of undernutrition in hospitalised patients. Nurs Stand. 2007;22(12):35-8. doi: 10.7748/ns2007.11.22.12.35.c6242. [PubMed: 18087876].

13. Muscaritoli M, Anker SD, Argiles J, Aversa Z, Bauer JM, Biolo G, et al. Consensus definition of sarcopenia, cachexia and pre-cachexia: Joint document elaborated by special interest groups (SIG) "cachexiaanorexia in chronic wasting diseases" and "nutrition in geriatrics". Clin Nutr. 2010;29(2):154-9. doi: 10.1016/j.clnu.2009.12.004. [PubMed: 20060626].

14. Pirlich M, Schutz T, Norman K, Gastell S, Lubke HJ, Bischoff SC, et al The German hospital malnutrition study. Clin Nutr. 2006;25(4):56372. doi: 10.1016/j.clnu.2006.03.005. [PubMed:16698132].

15. O'Flynn J, Peake H, Hickson M, Foster D, Frost G. The prevalence of malnutrition in hospitals can be reduced: Results from three consecutive cross-sectional studies. Clin Nutr. 2005;24(6):1078-88. doi: 10.1016/j.clnu.2005.08.012. [PubMed: 16219393].

16. Stratton RJ, King CL, Stroud MA, Jackson AA, Elia M. 'Malnutrition Universal Screening Tool' predicts mortality and length of hospital stay in acutely ill elderly. BrJ Nutr. 2006;95(2):325-30. [PubMed: 16469149]

17. Garcia RS, Tavares LR, Pastore CA. Nutritional screening in surgical patients of a teaching hospital from southern Brazil: The impact of nutritional risk in clinical outcomes. Einstein (Sao Paulo). 2013;11(2):14752. [PubMed: 23843052]. [PubMed Central: PMC4872885].

18. Bishop CW, Bowen PE, Ritchey SJ. Norms for nutritional assessment of American adults by upper arm anthropometry. Am J Clin Nutr. 1981;34(11):2530-9. doi:10.1093/ajcn/34.11.2530. [PubMed: 6975563]. 
19. Chakravarty C, Hazarika B, Goswami L, Ramasubban S. Prevalence of malnutrition in a tertiary care hospital in India. Indian J Crit Care Med. 2013;17(3):170-3. doi: 10.4103/0972-5229.117058. [PubMed: 24082614]. [PubMed Central: PMC3777371].

20. Stratton RJ, Hackston A, Longmore D, Dixon R, Price S, Stroud M, et al. Malnutrition in hospital outpatients and inpatients: Prevalence, concurrent validity and ease of use of the 'malnutrition universal screening tool' ('MUST') for adults. Br J Nutr. 2004;92(5):799-808. [PubMed: 15533269].

21. Mueller C, Compher C, Ellen DM; American Society for Parentera and Enteral Nutrition (A.S.P.E.N.) Board of Directors. A.S.P.E.N. clinical guidelines: Nutrition screening, assessment, and intervention in adults. JPEN J Parenter Enteral Nutr. 2011;35(1):16-24. doi 10.1177/0148607110389335. [PubMed: 21224430].

22. Gout BS, Barker LA, Crowe TC. Malnutrition identification, diagnosis and dietetic referrals: Are we doing a good enough job? Nutr Diet 2009;66(4):206-11. doi: 10.1111/j.1747-0080.2009.01372.x.

23. Edington J, Boorman J, Durrant ER, Perkins A, Giffin CV, James R, et al. Prevalence of malnutrition on admission to four hospitals in England. The Malnutrition Prevalence Group. Clin Nutr. 2000;19(3):191-5. doi:10.1054/clnu.1999.0121. [PubMed: 10895110].

24. Bharadwaj S, Ginoya S, Tandon P, Gohel TD, Guirguis J, Vallabh H, et al. Malnutrition: Laboratory markers vs nutritional assessment Gastroenterol Rep (Oxf). 2016;4(4):272-80. doi: 10.1093/gastro/gow013. [PubMed: 27174435]. [PubMed Central: PMC5193064]

25. Splett PL, Roth-Yousey LL, Vogelzang JL. Medical nutrition therapy for the prevention and treatment of unintentional weight loss in residential healthcare facilities. J Am Diet Assoc. 2003;103(3):352-62. doi: 10.1053/jada.2003.50050. [PubMed:12616259].

26. Kimiagar SM, Bazhan M. [Poverty and malnutrition in Iran]. Social Welfare. 2005;5(18):91-111. Persian.

27. Imoberdorf R, Meier R, Krebs P, Hangartner PJ, Hess B, Staubli M, et al. Prevalence of undernutrition on admission to Swiss hospitals. Clin Nutr. 2010;29(1):38-41. doi: 10.1016/j.clnu.2009.06.005. [PubMed: 19573958].

28. Fanian H, Pahlavansabagh A, Azarman N, Nahid A. Prevalence of malnutrition in orthopedic patients in Al-Zahra Hospital, Isfahan, Iran.J Res Med Sci. 2004;9(2):102.

29. Feldblum I, German L, Castel H, Harman-Boehm I, Bilenko N, Eisinger $\mathrm{M}$, et al. Characteristics of undernourished older medical patients and the identification of predictors for undernutrition status. Nutr J. 2007;6:37. doi: 10.1186/1475-2891-6-37. [PubMed: 17980023]. [PubMed Central: PMC2204029].

30. Kruizenga HM, Seidell JC, de Vet HC, Wierdsma NJ, van Bokhorstde van der Schueren MA. Development and validation of a hospital screening tool for malnutrition: The short nutritional assessment questionnaire (SNAQ). Clin Nutr. 2005;24(1):75-82. doi: 10.1016/j.clnu.2004.07.015. [PubMed: 15681104].

31. Elia M, Zellipour L, Stratton RJ. To screen or not to screen for adult malnutrition? Clin Nutr. 2005;24(6):867-84. doi: 10.1016/j.clnu.2005.03.004. [PubMed: 15893857]. 